\title{
Cross-species amplification of microsatellites and identification of polyploid hybrids by allele dosage effects in Cobitis hankugensis $\times$ Iksookimia longicorpa hybrid complex (Actinopterygii: Cypriniformes: Cobitidae)
}

\author{
Seung-Woon YUN ${ }^{1,2}$, Jong-Young PARK ${ }^{1}$, Karel JANKO ${ }^{2,3}$ \\ 1 Faculty of Biological Sciences and Institute for Biodiversity Research, Chonbuk National University, South Korea \\ 2 Laboratory of Fish Genetics, Institute of Animal Physiology and Genetics, Academy of Sciences of Czech Republic, Czech Republic \\ 3 Department of Biology and Ecology, Faculty of Natural Sciences, University of Ostrava, Czech Republic \\ http://zoobank.org/D3C2AAED-4D36-47DA-8A62-4F2023EF52EF \\ Corresponding author: Seung-Woon Yun (sjaksysw@hanmail.net); Karel Janko (janko@iapg.cas.cz)
}

Academic editor: Jan Kotusz • Received 24 October 2018 • Accepted 20 January 2021 • Published 12 July 2021

Citation: Yun S-W, Park J-Y, Janko K (2021) Cross-species amplification of microsatellites and identification of polyploid hybrids by allele dosage effects in Cobitis hankugensis $\times$ Iksookimia longicorpa hybrid complex (Actinopterygii: Cypriniformes: Cobitidae). Acta Ichthyologica et Piscatoria 51(2): 167-174. https://doi.org/10.3897/aiep.51.63591

\begin{abstract}
During the course of evolution, numerous taxa abandoned canonical sex and reproduced asexually. Examination of the Cobitis hankugensis $\times$ Iksookimia longicorpa asexual complex already revealed important evolutionary discoveries tackling phenomena like interspecific hybridization, non-Mendelian inheritance, polyploidy, and asexuality. Yet, as in other similar cases, the investigation is hampered by the lack of easily accessible molecular tools for efficient differentiation among genomotypes. Here, we tested the cross-species amplification of 23 microsatellite markers derived from distantly related species and investigated the extent to which such markers can facilitate the genome identification in the non-model hybrid complex. We found that 21 out of 23 microsatellite markers were amplified in all genomotypes. Five of them could be used for easy diagnostics of parental species and their hybrids due to species-specific amplification profiles. We also noted that three markers, i.e., IC654 and IC783 derived from Cobitis choii Kim et Son, 1984 and Iko TTA01 from Iksookimia koreensis (Kim, 1975), had dosage-sensitive amplification efficiencies of species-specific alleles. This could be further used for reliable differentiation of genome composition in polyploids. The presently reported study introduces a noninvasive method applicable for the diagnosis of ploidy and genome composition of hybrids, which are not clearly distinguished morphologically. We showed that very detailed information may be obtained even from markers developed in distantly related taxa. Hybridization is being increasingly recognized as a driving force in evolution. Yet, proper detection of hybrids and their ploidy is particularly challenging, especially in non-model organisms. The present paper evaluates the power of microsatellite cross-amplification not only in the identification of hybrid forms but also in estimating their genome dosage on an example of a fish taxon that involves asexuality, hybridization as well as ploidy variation. It thus demonstrates the wide applicability of such cheap and non-invasive tools.
\end{abstract}

\section{Keywords}

Microsatellites, cross-amplification, Iksookimia longicorpa $\times$ Cobitis hankugensis complex, hybridization, asexual reproduction, polyploidy

Copyright Seung-Woon Yun et al. This is an open access article distributed under the terms of the Creative Commons Attribution License (CC BY 4.0), which permits unrestricted use, distribution, and reproduction in any medium, provided the original author and source are credited. 


\section{Introduction}

Although initially neglected in zoological literature, hybridization and polyploidy are attracting considerable research interest as mighty evolutionary mechanisms. Both phenomena are further linked with aberrant reproductive modes leading to the so-called asexual lineages with more or less severe deviations from canonical Mendelian reproduction (Janko et al. 2018). The order Cypriniformes represents a diverse group of primarily freshwater fish where incidences of hybridization, polyploidy, and asexuality are relatively frequent and new cases are still being discovered in recent times ( $\mathrm{Li}$ et al. 2014). The group divides into several lineages of which one, the suborder Cyprinoidei, is heavily exploited and explored for both scientific and commercial purposes. Unlike Cyprinoidei, its sister lineage Cobitoidea remains largely understudied although it represents a very speciose group. Only recently has the proper taxonomy of this group been investigated, which lead to discoveries of new species (Janko et al. 2005) and even entirely new families (Bohlen and Šlechtová 2009). The group Cobitiodei is adapted to almost every water habitat ranging from standing anoxic waters to high mountain streams and contains several extravagant cases of independently evolved hybrid complexes (including the intergeneric gene exchange (Šlechtová et al. 2008), polyploidizations and asexually reproducing lineages (Kim and Lee 2000; Janko et al. 2007). The reason why this group remains relatively poorly studied lays in its benthic lifestyle promoting conservativeness of body shape and the consequent presence of many cryptic species, which may be diagnosed only by molecular markers (e.g., Janko et al. 2005). This study focuses on a South Korean member of the suborder, the so-called Cobitis hankugensis $\times$ Iksookimia longicorpa hybrid complex, which contains two hybridizing species and a wide array of asexual diploid and polyploid hybrid lineages, which will also be referred to as genomotypes in the subsequent text. In particular, we describe new molecular tools for the proper determination of those forms, which will streamline future research of this model taxon.

The hybridization between Cobitis hankugensis and its confamilial relative Iksookimia longicorpa was first reported at the Nakdong River tributaries by Kim and Lee
(1990) who documented the existence of di- and triploid all-female hybrid population. Later, Lee (unpublished thesis) reported that ecological and morphological traits of hybrids appear intermediate between their parental species. Based on the results of chromosome analysis, Lee (unpublished thesis) revealed that hybrids consist of two genomotypes, namely diploid hankugensis $\times$ longicorpa (hereafter HL, where the letter L stands for longicorpa genome whereas the letter $\mathrm{H}$ stands for hankugensis genome) and triploid with two hankugensis and one longicorpa genomes (hereafter HHL). Additionally, Lee (1995) reported another triploid form with one hankugensis and two longicorpa genomes (hereafter HLL). Kim and Lee (2000) and Ko (2009) documented an exceptional way of reproduction in these hybrids where diploid females produce unreduced eggs, which accept the sperm of males from one of the parental species and give rise to one or the other type of triploids. Triploid females exclude the entire genome of the parental species contributing to the haploid chromosomal set and subsequently upon meiotic divisions of the remaining two chromosomal sets produce a haploid egg, which, if fertilized by a male of the former species may give rise to diploid clone again. Such diploid and triploid generation alternation has never been described in any other asexual fish to date, making this taxon an outstanding model for the research of aberrant reproductive modes. The summary of known reproductive interactions is provided in Fig. 1.

Although studies of this complex may bring discoveries of general importance, they are complicated by nontrivial morphological identification of the three types of hybrids. Cytological and molecular biological approaches are therefore required for their accurate discrimination. However, while diploid and triploid forms may be easily discriminated through the measurement of erythrocyte cell size or by flow cytometry, the two types of triploids may not be discriminated by the flow cytometry due to the absence of significant differences in DNA content between the parental species. Chromosomal counting thus remains the most reliable differentiation method to date, but it has a fatal disadvantage of being extremely timely and invasive. For these reasons, a new approach is needed for further study of the hybrid complex.

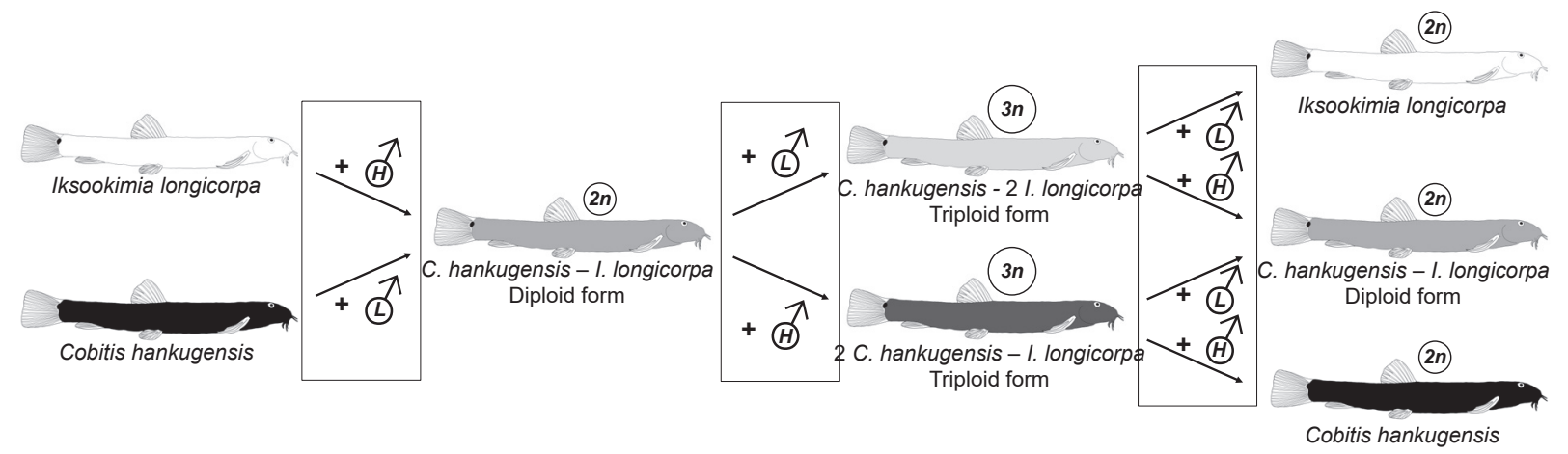

Figure 1. The summary of known reproductive interactions between parental species and hybrid forms of the Cobitis hankugensis $\times$ Iksookimia longicorpa hybrid complex. 
Microsatellite loci analysis, one of the most widely used molecular biology research methods, is an accurate tool for verifying genealogy and identification of relatives, as well as demonstrating genetic diversity by separating and analyzing markers that are inherent in each chromosome (McConnell et al. 1995; Nelson et al. 1998; Smith et al. 1998; Goldstein and Schlötterer 1999; Beacham et al. 2000; Sunnucks 2000; Tian et al. 2017). To date, the microsatellite markers have been developed for several species of the family Cobitidae, including Cobitis taenia Linnaeus, 1758 (see de Gelas et al. 2008), C. choii (see Bang et al. 2009), Iksookimia koreensis (see Yu et al. 2014), and Koreocobitis naktongensis Kim, Park et Nalbant, 2000 (see Anonymous 2011a). However, the development of microsatellite markers targeting C. hankugensis, I. longicorpa, and C. hankugensis $\times I$. longicorpa hybrid has not yet been reported. While the de novo development of microsatellite markers is labor and cost intensive (Zane et al. 2002; Squirrell et al. 2003; Thiel et al. 2003; Gonzalez-Martinez et al. 2004; Senan et al. 2014), the cross-amplification using the already identified markers is relatively easily accessible. Therefore, in this study, we apply the cross-amplification of markers previously developed microsatellite for related Cobitis species on the $C$. hankugensis $\times I$. longicorpa hybrid complex with the special aim to discriminate among all hybrid genomotypes including the two types of triploids.

\section{Materials and methods}

\section{Sample collection and identification}

Sampled fish were treated according to the "Ethical justification for the use and treatment of fishes in research" (Anonymous 2006). This study was carried out in strict accordance with the recommendations in the Guide for the care and use of laboratory animals of the National Institutes of Health (Anonymous 2011b). The fish dissection was performed under MS-222 anesthesia, and all efforts were made to minimize the pain. The collection of I. longicorpa, C. hankugensis, and their hybrids was carried out in three areas with several localities (Fig. 2, Table 2), i.e., in the Seomjin River basin, Imsil, Jeollabuk-do, where I. longicorpa occurs, in the Nakdong River basin, Hapcheon, Gyeongsangnam-do, where C. hankugensis occurs and in the Nakdong River basin, Namwon, Jeollabuk-do.

The identification of collected specimens was based on previously published methods. In particular, we examined each specimen by morphology, which is known to consistently distinguish both parental species from each other as well as their hybrids (albeit, we stress that morphological analysis may not reliably distinguish among different genomotypes of hybrids) (Kim and Park 2002). The ploidy was evaluated by erythrocyte size measurement (Ko 2009). To cross-validate our determination and to precisely evaluate the genomic composition, we employed karyotype analysis to a subset of specimens. This method

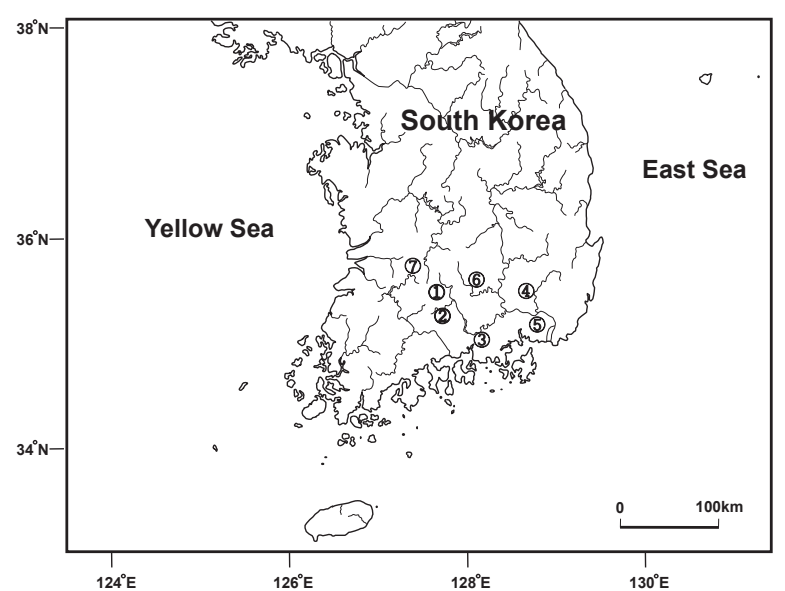

Figure 2. Sampling localities of Cobitis hankugensis $\times$ Iksookimia longicorpa hybrid complex used in this study.

provides reliable determination of sexual or hybrid genomotype given that both sexual species differ by chromosomal numbers (I. longicorpa $2 \mathrm{n}=50 ;$ C. hankugensis $2 n=48$ ), therefore allowing easy determination of both parental species (hereafter also labeled as LL and HH, respectively) from their diploid (HL, $2 \mathrm{n}=49$ ) and triploid (HHL $3 n=73$ or HLL $3 n=74$ ) hybrid forms (Fig. 3 ).

Finally, to obtain comparative material with known origin and genome composition, we have also performed 4 experimental crosses of parental species to obtain strict F1 HL hybrids, and we also crossed natural diploid HL hybrid females with either LL (3 families) or HH (3 families) males to obtain triploid HHL and HLL hybrids, altogether yielding a total of 133 experimental progeny of verified origin for microsatellite genotyping.

\section{Microsatellite marker selection}

For the cross-species amplification analysis, the aforementioned fish samples were scrutinized for previously published microsatellite markers developed for related species of the Cobitidae family. The list of loci is shown in Table 1.

\section{DNA Amplification and Genotyping}

For DNA analysis a piece of pectoral fin was dissected from each specimen and stored in $100 \%$ ethyl alcohol. Total DNA was purified with the genomic DNA Prep Kit for blood and tissue (QIAGEN Co., USA). PCR reactions were completed in a total volume of $50 \mu \mathrm{L}$, consisting of $2 \mu \mathrm{L}$ of genomic DNA, $1 \mu \mathrm{L}$ of the $10 \mathrm{uM}$ forward (fluorescently labeled) and reverse primer solutions, 24 $\mu \mathrm{L}$ of Premix Taq (Takara, Japan), and $22 \mu \mathrm{L}$ of distilled water (Takara, Japan). Polymerase chain reactions for all specimens were executed in GeneAtlas G-02 thermocycler (Astec, Japan) with the initial denaturing step at $95^{\circ} \mathrm{C}$ for $5 \mathrm{~min}$ and 35 cycles of $30 \mathrm{~s}$ at $94^{\circ} \mathrm{C}, 30 \mathrm{~s}$ at $55^{\circ} \mathrm{C}$, and 
Table 1. Details of 23 microsatellite markers used for Cobitis hankugensis $\times$ Iksookimia longicorpa hybrid detection. Cross-amplification results are indicated for both Iksookimia longicorpa, Cobitis hankugensis species. Five markers with suited properties for genomotype identification and dosage effects are highlighted in bold.

\begin{tabular}{|c|c|c|c|c|c|c|c|c|c|c|c|}
\hline \multirow[t]{2}{*}{ Locus } & \multirow{2}{*}{\multicolumn{2}{|c|}{ Primer sequence $\left(5^{\prime} \rightarrow 3^{\prime}\right)$}} & \multirow[t]{2}{*}{$\begin{array}{c}\text { Repeat } \\
\text { motif }\end{array}$} & \multirow[t]{2}{*}{ Reference species } & \multirow[t]{2}{*}{$\begin{array}{l}\text { Accession } \\
\text { No. }\end{array}$} & \multicolumn{2}{|c|}{$\begin{array}{l}\text { Reference } \\
\text { species }\end{array}$} & \multicolumn{2}{|c|}{$\begin{array}{l}\text { Iksookimia } \\
\text { longicorpa }\end{array}$} & \multicolumn{2}{|c|}{$\begin{array}{c}\text { Cobitis } \\
\text { hankugensis }\end{array}$} \\
\hline & & & & & & $N_{A}$ & $\begin{array}{c}\text { Allele } \\
\text { size }[b p]\end{array}$ & $N_{\mathrm{A}}{ }^{*}$ & $\begin{array}{c}\text { Allele } \\
\text { size [bp] }\end{array}$ & $N_{\mathrm{A}}$ & $\begin{array}{c}\text { Allele } \\
\text { size [bp] }\end{array}$ \\
\hline Cota_006 & $\begin{array}{l}\text { F: } \\
\text { R: }\end{array}$ & $\begin{array}{l}\text { HEX-GCAGGTACAGAACCCCGACATGG } \\
\text { AGTACGGCCCTATGGGGTTTGAC }\end{array}$ & TTG/CTAT & $\begin{array}{c}\text { Cobitis taenia } \\
\text { (see de Gelas et al. } \\
\text { 2008) }\end{array}$ & EU276579 & 11 & $336-374$ & 2 & $163-165$ & 2 & $163-165$ \\
\hline Cota_025 & $\begin{array}{l}\text { F: } \\
\text { R: }\end{array}$ & $\begin{array}{c}\text { 6-FAM-TGCGTTTACAAGATTCGTGTGGAC } \\
\text { GCTGCATATGAGTAACATGTCTG }\end{array}$ & CACG & & EU276580 & 3 & $144-160$ & 2 & $42-52$ & 2 & $42-52$ \\
\hline Cota_032 & $\begin{array}{l}\text { F: } \\
\text { R: }\end{array}$ & $\begin{array}{c}\text { 6-FAM-TGGTCATGACTGGCACACCGTC } \\
\text { AGGAGGTTTGAAGAAGGGCAAG }\end{array}$ & TCTT & & EU276582 & 2 & $232-236$ & 2 & $271-290$ & 4 & $271-301$ \\
\hline Cota_033 & $\begin{array}{l}\text { F: } \\
\text { R: }\end{array}$ & $\begin{array}{l}\text { HEX-TTTCTGAATCAAGAGCCCAGCAGT } \\
\text { AGATATGACATCCAATCACACGCT }\end{array}$ & AGAC & & EU276583 & 3 & $211-235$ & 2 & $203-207$ & 1 & 207 \\
\hline Cota_037 & $\begin{array}{l}\text { F: } \\
\text { R: }\end{array}$ & $\begin{array}{l}\text { 6-FAM-GCACTCGAGTCGATTCGGTGGCGC } \\
\text { GTAATCAATCAGTCCAAAGCACTT }\end{array}$ & GA & & EU276584 & 3 & $272-276$ & 6 & $275-304$ & 4 & $280-298$ \\
\hline Cota_093 & $\begin{array}{l}\text { F: } \\
\text { R: }\end{array}$ & $\begin{array}{c}\text { 6-FAM-CCCTGGGAGTTCTCAGCAGGACTG } \\
\text { ATAATGCACATTGTTGGGCTGC }\end{array}$ & $\mathrm{AC}$ & & EU276586 & 4 & $341-357$ & 1 & 304 & 1 & 304 \\
\hline IC248 & $\begin{array}{l}\text { F: } \\
\text { R: }\end{array}$ & $\begin{array}{c}\text { HEX-CACTCTGAGGCGAAACTGGAG } \\
\text { TCAAATCATATAGTGCAGCCAAGC }\end{array}$ & $\mathrm{CA}$ & $\begin{array}{c}\text { Cobitis choii } \\
\text { (see Bang et al. 2009) }\end{array}$ & EU252088 & 24 & $123-187$ & 6 & $117-148$ & 4 & $107-119$ \\
\hline $\mathrm{IC} 252$ & $\begin{array}{l}\text { F: } \\
\text { R: }\end{array}$ & $\begin{array}{l}\text { HEX-AATGAGACGGGTAACTTGTGTATG } \\
\text { GCTGATCTATGATTGGTTGTGTC }\end{array}$ & $\mathrm{CA}$ & & EU252089 & 12 & $188-218$ & 1 & 155 & 1 & 155 \\
\hline IC276 & $\begin{array}{l}\text { F: } \\
\text { R: }\end{array}$ & $\begin{array}{l}\text { 6-FAM-GTAACTCCGGGCGTGTAACTCTG } \\
\text { CACTGTAGAACCCAGCCAAAACC }\end{array}$ & GT & & EU252090 & 14 & $82-114$ & 1 & 70 & 1 & 70 \\
\hline IC372 & $\begin{array}{l}\text { F: } \\
\text { R: }\end{array}$ & $\begin{array}{c}\text { 6-FAM-ACACGCACACCTATTACAACCTA } \\
\text { GATTTGCCAGTGTGCTAATTG }\end{array}$ & $\mathrm{AC}$ & & EU252091 & 33 & $77-169$ & 2 & $86-90$ & 2 & $86-90$ \\
\hline IC434 & $\begin{array}{l}\text { F: } \\
\text { R: }\end{array}$ & $\begin{array}{l}\text { 6-FAM-TCCACCATGACCATTTTTACATA } \\
\text { GGTGTCTGGATCTCATCTTGAA }\end{array}$ & $\mathrm{AC}$ & & EU252092 & 23 & $83-165$ & 1 & 78 & 1 & 78 \\
\hline IC645 & $\begin{array}{l}\text { F: } \\
\text { R: }\end{array}$ & $\begin{array}{c}\text { 6-FAM-CTCTGAGACAACTCGGTAGTCCC } \\
\text { CACATACATGGCCTGCAACAT }\end{array}$ & $\mathrm{CA}$ & & EU252095 & 19 & $161-225$ & 1 & 189 & 1 & 189 \\
\hline IC654 & $\begin{array}{l}\text { F: } \\
\text { R: }\end{array}$ & $\begin{array}{c}\text { HEX-TGAGCCGACACTAGAAACAGAGC } \\
\text { GACAAAGTGCAGGCACAGAATG }\end{array}$ & CA & & EU252096 & 14 & $158-208$ & 1 & 130 & 1 & 138 \\
\hline IC783 & $\begin{array}{l}\text { F: } \\
\text { R: }\end{array}$ & $\begin{array}{c}\text { HEX-GGAGAAGATGTGATGGAGATG } \\
\text { ATATTATGATGGGAAGACACGAC }\end{array}$ & AC & & EU252098 & 22 & $146-196$ & 2 & $120-123$ & 1 & 127 \\
\hline IC839 & $\begin{array}{l}\text { F: } \\
\text { R: }\end{array}$ & $\begin{array}{l}\text { 6-FAM-TTGTTCCCCTCTGAAACCCAATC } \\
\text { GTGTTAGCCCGTGTGCCAAAG }\end{array}$ & $\mathrm{CA}$ & & EU252100 & 13 & $99-125$ & 5 & $92-110$ & 5 & $82-94$ \\
\hline IC875 & $\begin{array}{l}\text { F: } \\
\text { R: }\end{array}$ & $\begin{array}{c}\text { HEX-AGCGGTGTGGATGTGAATGCTAA } \\
\text { CTTGTCAGGCTCTGGCACTCG }\end{array}$ & CA & & EU252101 & 22 & $132-182$ & & - & 9 & 134-158 \\
\hline Iko_AAT08 & $\begin{array}{l}\text { F: } \\
\text { R: }\end{array}$ & $\begin{array}{l}\text { 6-FAM-GTGATGCAAATGTCTTCTGTGT } \\
\text { CAAATCTTTCCTTTGTCTTTGG }\end{array}$ & ATT & $\begin{array}{l}\text { Iksookimia koreensis } \\
\text { (see Yu et al. 2014) }\end{array}$ & KJ588473 & 5 & $147-163$ & 2 & $125-135$ & & - \\
\hline Iko_TTA01 & $\begin{array}{l}\text { F: } \\
\text { R: }\end{array}$ & $\begin{array}{c}\text { 6-FAM-ACATTAGTGGGGTAAGATGTGC } \\
\text { AAGGAAGGAATAGGGTAAGCTG }\end{array}$ & TTA & & KJ588474 & 8 & $180-238$ & 1 & 321 & 1 & 330 \\
\hline KN03 & F: & TGATATCATCGGTGTAAATGTTTAAGA & $\mathrm{CA}$ & $\begin{array}{l}\text { Koreocobitis nakton- } \\
\text { genesis } \\
\text { (see Anonymous 2011) }\end{array}$ & JN203057 & 8 & $134-156$ & 1 & 116 & 1 & 116 \\
\hline KN16 & $\begin{array}{l}\text { F: } \\
\text { R: }\end{array}$ & $\begin{array}{l}\text { HEX-CGACGTAGAGTCAAAAGTGCG } \\
\text { TGGAGATCAGGTTACGGGTG }\end{array}$ & $\mathrm{CA}$ & & JN203058 & 10 & $135-157$ & 1 & 126 & 1 & 126 \\
\hline KN20 & $\begin{array}{l}\text { F: } \\
\text { R: }\end{array}$ & $\begin{array}{l}\text { HEX-TTGTGCTGATAACACATCCTGC } \\
\text { GATTGAATCATCCGCAGAGC }\end{array}$ & $\mathrm{CA}$ & & JN203059 & 10 & $144-172$ & 1 & 137 & 1 & 137 \\
\hline KN25 & $\begin{array}{l}\mathrm{F}: \\
\mathrm{R}:\end{array}$ & $\begin{array}{c}\text { 6-FAM-CGTTCCCCTCAGGTCTCAAT } \\
\text { CCTGCAGTTTTCAGCCAAGA }\end{array}$ & $\mathrm{CA}$ & & JN203060 & 9 & $275-295$ & 4 & $293-313$ & 4 & $307-313$ \\
\hline KN34 & $\begin{array}{l}\text { F: } \\
\text { R: }\end{array}$ & $\begin{array}{c}\text { 6-FAM-CCAGTGGACATCTGCAACAAC } \\
\text { GCCCTGCTAGTGAGGAACAA }\end{array}$ & TG & & JN203062 & 9 & $273-289$ & 1 & 286 & 1 & 286 \\
\hline
\end{tabular}

${ }^{*} N_{\mathrm{A}}=$ number of alleles.

$1 \mathrm{~min}$ at $72^{\circ} \mathrm{C}$. A final extension step at $72^{\circ} \mathrm{C}$ for $5 \mathrm{~min}$. The PCR amplicons were visualized on a $2 \%$ agarose gel stained with LoadingStar (Dyne, Korea) together with negative controls and Takara $1 \mathrm{~KB}$ molecular size ladder for preliminary size determination. The final PCR products were run on an ABI-3730XL sequencer (Applied Biosystems, USA) with the size standard at 350 ROX. The resulting electropherograms were analyzed in Peak Scanner v1.0 (Applied Biosystems, USA).

To evaluate whether particular markers bear consistent information about the allelic dosage in diploid and triploid hybrids, we used the Gene scan peak analysis with the Peak Scanner v1.0 (Applied Biosystems, USA) to analyze and compare the relative intensities of alleles in analyzed individuals.

\section{Results and discussion}

\section{Determination of experimental animals with classical markers}

Altogether, based on the classical determination methods including karyotype analysis we selected for marker vali- 
A

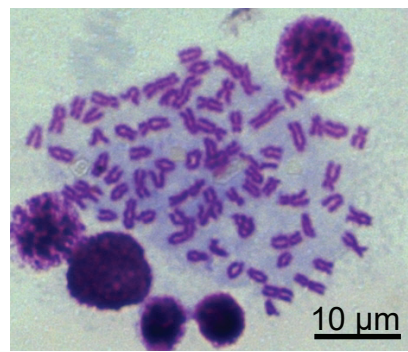

B

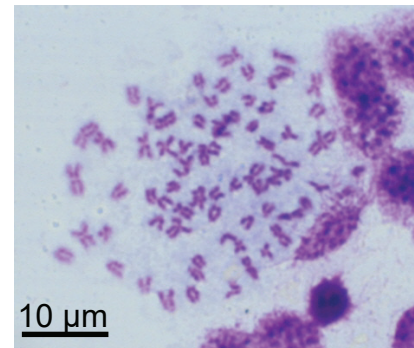

\begin{tabular}{|c|c|c|c|c|c|c|c|c|c|c|c|c|c|c|c|c|c|c|c|}
\hline & 8 & 8 & 8 & 8 & 8 & 8 & 8 & 8 & $8^{10}$ & $\gamma$ & 8 & 8 & $x$ & 8 & 8 & $\approx$ & $\gamma$ & $x$ & 8 \\
\hline & $\gamma$ & $x$ & 8 & 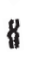 & $\gamma$ & 1 & 8 & 8 & $\gamma^{20}$ & $x$ & $\pi$ & $x$ & $x$ & $x$ & 8 & $x$ & X & $x$ & $\gamma^{20}$ \\
\hline & $\gamma$ & $x$ & $x$ & $\not$ & " & $x$ & $x$ & $x$ & $x^{30}$ & $x$ & $x$ & x & $*$ & $x$ & * & * & 8 & $n$ & $a^{30}$ \\
\hline & $n$ & 0 & $n$ & $n$ & 0 & 8 & $a$ & 1 & (1) 40 & $n$ & $a$ & A & ภ & $n$ & 0 & A & $n$ & $n$ & $n^{40}$ \\
\hline & 0 & 8 & i) & $A$ & 8 & 0 & 2 & $R$ & $8^{50}$ & $n$ & $n$ & n & 1 & 8 & $n$ & $\lambda$ & ^ & $n$ & $n^{50}$ \\
\hline & A & $A$ & 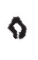 & $\Omega$ & 0 & 0 & 0 & $n$ & $R^{60}$ & a & A & ^ & $\pi$ & n & $n$ & n & $n$ & $n$ & $n^{60}$ \\
\hline & 8 & $n$ & is & $n$ & $\wedge$ & $\Omega$ & $n$ & ^ & $\Delta^{70}$ & ^ & n & A & $\wedge$ & a & A & A & ^ & n & م $^{70}$ \\
\hline & & $\alpha$ & & & & & & & & ح & - & $\sim$ & & & & & & & \\
\hline
\end{tabular}

Figure 3. Metaphase plates and karyotypes of two triploid Cobitis hankugensis $\times$ Iksookimia longicorpa hybrids. A: genotype HLL; B: genotype HHL.

Table 2. Study localities of Cobitis hankugensis $\times$ Iksookimia longicorpa hybrid complex used in this study.

\begin{tabular}{lccc}
\hline No. & River basin & Locality & Coordinates \\
\hline 1 & Ram Stream & Inwol-myeon, Namwon-si, Jeollabuk-do & $35^{\circ} 27^{\prime} 27.2^{\prime \prime} \mathrm{N}, 127^{\circ} 36^{\prime} 25.6^{\prime \prime} \mathrm{E}$ \\
2 & Nam River & Saengcho-myeon, Sancheong-gun, Gyeongsangnam-do & $35^{\circ} 28^{\prime} 46.9^{\prime \prime} \mathrm{N}, 127^{\circ} 50^{\prime} 56.9^{\prime \prime} \mathrm{E}$ \\
3 & Banseong Stream & Ibanseong-myeon, Jinju-si, Gyeongsangnam-do & $35^{\circ} 9^{\prime} 51.8^{\prime \prime} \mathrm{N}, 128^{\circ} 17^{\prime} 44.8^{\prime \prime} \mathrm{E}$ \\
4 & Cheongdo Stream & Punggak-myeon, Cheongdo-gun, Gyeongsangbuk-do & $35^{\circ} 38^{\prime} 37.2^{\prime \prime} \mathrm{N}, 128^{\circ} 37^{\prime} 25.8^{\prime \prime} \mathrm{E}$ \\
5 & Unjeong Stream & Muan-myeon, Miryang-si, Gyeongsangnam-do & $35^{\circ} 29^{\prime} 37.6^{\prime \prime} \mathrm{N}, 128^{\circ} 40^{\prime} 11.1^{\prime \prime} \mathrm{E}$ \\
6 & Hwanggye Stream & Yongju-myeon, Hapcheon-gun, Gyeongsangnam-do & $35^{\circ} 30^{\prime} 20.3^{\prime \prime} \mathrm{N}, 128^{\circ} 6^{\prime} 17.2^{\prime \prime} \mathrm{E}$ \\
7 & Oknyeodong Stream & Unam-myeon, Imsil-gun, Jeollabuk-do & $35^{\circ} 39^{\prime} 35.3^{\prime \prime} \mathrm{N}, 127^{\circ} 9^{\prime} 20.0^{\prime \prime} \mathrm{E}$ \\
\hline
\end{tabular}

dation 25 individuals of I. longicorpa, 25 of $C$. hankugensis, and also 5 HL, 5 HLL, and 5 HHL hybrid individuals. We further included into the analysis 59 natural hybrids (without karyotype analysis) sampled at five sites in the Nakdong River basin, and we also scrutinized 133 progenies generated by artificial crossing experiments with known origin and genomic composition.

\section{Cross-species amplification results and species diagnostics}

The cross-amplification of 23 published markers showed that 19 loci were amplified in all genomotypes of the hybrid complex. Moreover, we further noticed that the IC875 marker did not amplify with I. longicorpa but it did amplify in the C. hankugensis and hybrids while the Iko_AAT08 marker did not amplify at $C$. hankugensis, but it did amplify in the I. longicorpa and in hybrids (Table 1). We observed that $50.0 \%$ of tested loci were monomorphic in I. longicorpa, 59.1\% in C. hankugensis, and $43.5 \%$ in all three types of hybrids. The number of alleles per locus, except for monomorphic ones, ranged from 2-6 (mean 3.2) in I. longicorpa, 2-9 (mean 4.0) in C. hankugensis, 2-5 (mean 2.5) in hybrid (HL type), 2-4 (mean 2.9) in hybrid (HLL type), and 2-5 (mean 2.7) in hybrid (HHL type), respectively.
We note that in each locus, the numbers of detected alleles were always lower than those reported in the reference species for which given microsatellite marker has been developed and where 2-33 alleles per locus per species (mean 12.2) have been reported in the original publications. When compared to the reference species, analyzed hybrids had the highest numbers of alleles in markers taken from $C$. taenia (mean value 2.3 alleles per locus), the second-highest numbers of alleles in loci taken from $C$. choii (mean value 2.2), while the markers taken from different genera showed lowest numbers of alleles, i.e., mean value 1.5 in $K$. naktongensis markers and 1.4 in I. koreensis markers, respectively. This is in line with the general expectation that the efficiency of cross-species amplification tends to decrease with increasing phylogenetic distance between the reference species and the target species (Moore et al. 1991; Peakall et al. 1998). This study used the cross-amplification between species of the same genus or family and showed relatively high amplification efficiency. However, nearly half of essayed loci appeared as monomorphic and many of other loci shared the same alleles between species, making them of limited use for species diagnosis. Future studies of the genetic diversity of I. longicorpa and C. hankugensis and their hybrids would certainly profit from the direct development of microsatellite loci from their DNA. 
Nevertheless, we discovered three loci, which seem very useful for fast and efficient identification of genomotypes from the studied hybrid complex because they possess non-overlapping allelic size ranges between species. Specifically, the loci IC654, IC783, and Iko_TTA01 always distinguished between the specimens identified as pure I. longicorpa and C. hankugensis, respectively, while they always provided amplification products of both species in the hybrid individuals. Furthermore, we found two additional loci with selective amplification (IC875, Iko_AAT08), where one sexual species was characterized by absence of amplification, while the other species and all hybrid individuals provided specific amplification product (Table 1).

This altogether suggests that tested cross-amplification identified three markers with species-specific allelic variants and two loci with species-selective amplification that may be used as haploid detection markers for C. hankugensis and I. longicorpa, respectively. In addition, some other loci also appear as useful for subsequent population genetic studies given they possess a moderate number of alleles per species, which may allow for frequency-based analyses.

\section{Hybrid detection and allele dosage effects}

Given that the scope of this paper was to find a fast and efficient method to discern parental species and hybrid genomotypes, we will describe in the following text the properties of three markers that we propose for such a purpose given their ability to diagnose both species as well as the ploidy of hybrid individuals. The IC654 and IC783 markers derived from $C$. choii and the Iko_TTA01 marker derived from $I$. koreensis were of particular interest for us as they were fixed for different alleles in both parental species and showed the consistent presence of both species-specific amplification products in hybrids with different relative peak intensities depending on the genotypes (Fig. 3).

The patterns were straightforward in IC654 and Iko TTA01 markers (Fig. 4A-B) since in diploid hybrid HL genomotype with one genome of I. longicorpa and the other of $C$. hankugensis, the heights of the amplified peaks were similar to each other. In triploid hybrid HLL genomotype with two genomes of I. longicorpa and one of C. hankugensis, the allele peak intensity of I. longicorpa-
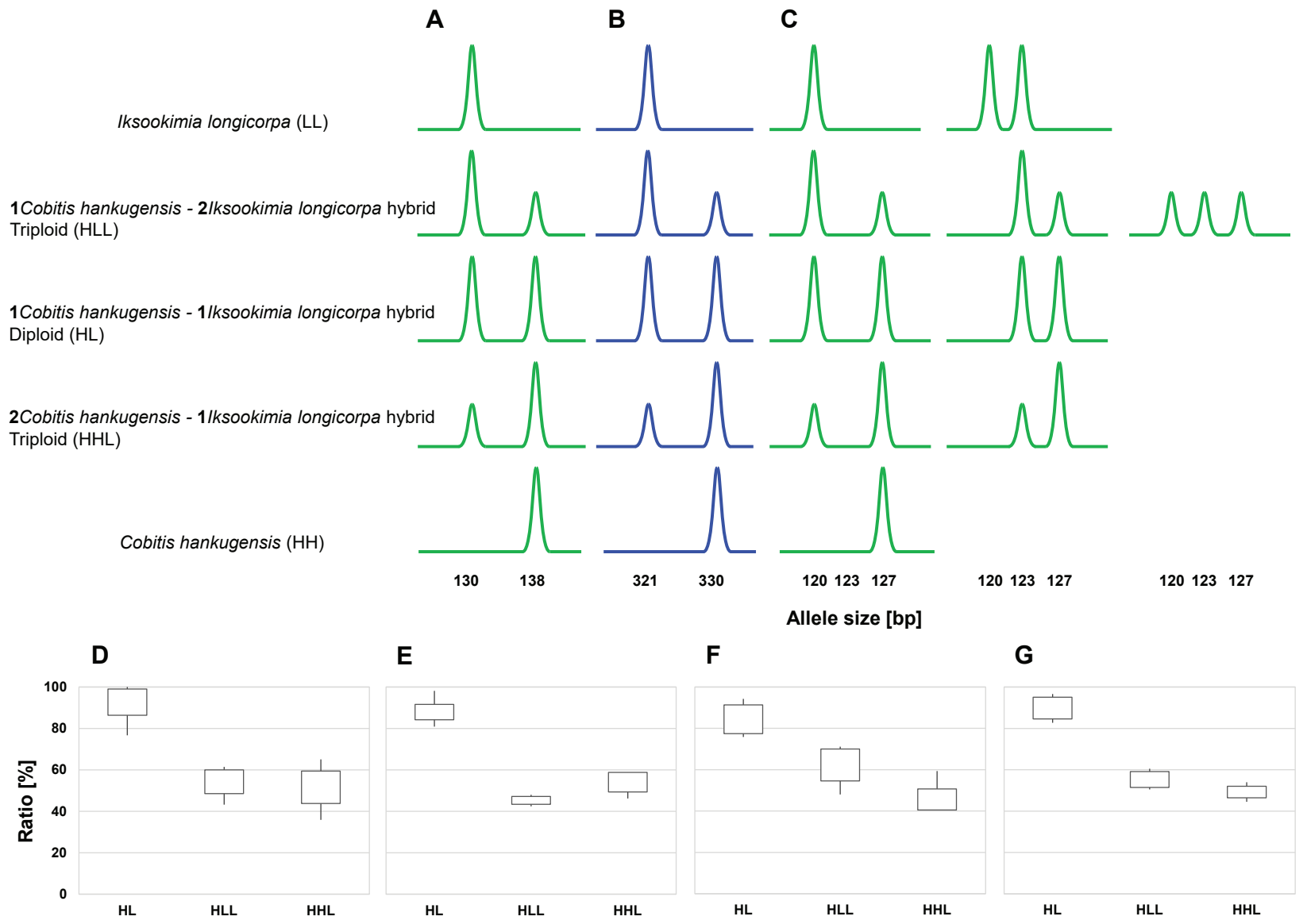

E

$\mathbf{F}$

G
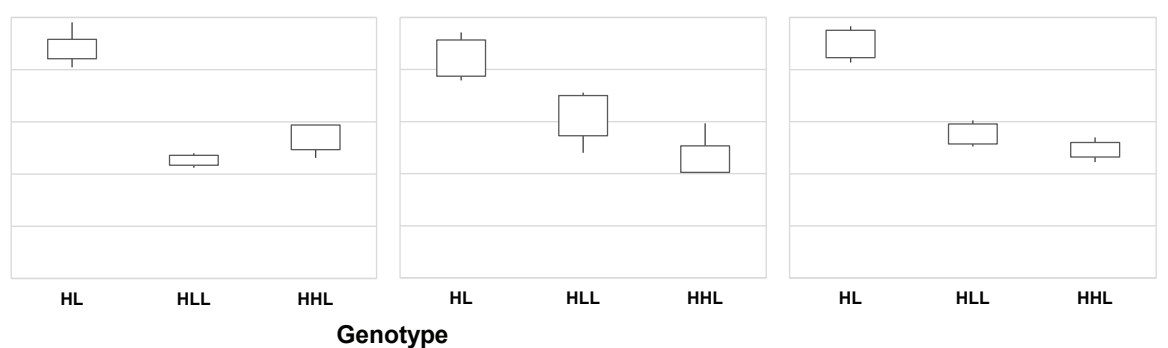

Figure 4. Demonstration of Microsatellite analysis of IC654 (A), Iko_TTA01 (B) and IC783 (C) loci in Cobitis hankugensis $\times$ Iksookimia longicorpa hybrid complex. Upper panels A-C show the electropherograms of the three loci in all biotypes. Boxplots in the lower panel depict for each locus the relative intensity (\%) of the minor peak to the major one (D-F) and the mean relative values of the total of three markers for each genomotype $(\mathbf{G})$. 
specific allele was approximately twice as strong as that of $C$. hankugensis - specific allele, while in triploid hybrid HHL genomotype the situation was opposite with approximately double intensity of the $C$. hankugensis allele compared to I. longicorpa allele. The patterns in IC783 were more complicated by the presence of two alleles in I. longicorpa. Consequently, the diploid HL form always possessed one allele diagnostic for I. longicorpa and the other for C. hankugensis, HHL triploids also possessed two alleles with clear dosage pattern, but HLL triploids either possessed two alleles with the apparent dosage pattern or three alleles, each with similar intensity (Fig. 4C).

To verify the possibility of applying the three selected markers (IC783, IC654, and Iko_TTA01) for the identification of unknown hybrid genomotype, we demonstrate the relative size ratio of the minor peak to the major one (Fig. 4 D-F). As a result, three hybrid genomotypes were clearly distinguished by the ratio of the peaks. Diploid HL genomotype had relatively similar intensities of the less intense allele, i.e., 92.7\% (IC654), 84.4\% (IC783), and 87.9\% (Iko TTA01), while in triploid HLL genomotype, the ratios were $54.3 \%, 62.3 \%$, and $45.3 \%$, respectively, and in HHL genomotype, we found $51.6 \%, 45.6 \%$, and $54.1 \%$, respectively (Fig. 4, D-F). Finally, we also plotted the mean relative values of the total of three markers for each genomotype, (Fig. $4, \mathrm{G})$, where the mean value in HL type was $89.8 \%$, in HLL type was $55.3 \%$, HHL type was $49.2 \%$. This result strongly supported that the microsatellite marker can be used to the correct method of discrimination of known genomotypes of the $C$. hankugensis $\times I$. longicorpa complex.

To date, the identification of $C$. hankugensis $\times$ I. longicorpa hybrid complex had a fatal disadvantage in that it re- quires complex processing and fish sacrifice. In this study, we provided a reliable identification method of the $C$. hankugensis $\times I$. longicorpa hybrid complex using microsatellite markers through a single Genescan analysis using only a small piece of fin tissues. This has the great advantage that the fish are kept alive and can be used for additional hybridization experiments by reducing the stress.

Microsatellite markers have indeed been previously used to identify hybrid groups of fish, including the family Cobitidae, (e.g., You et al. 2007; de Gelas et al. 2008) and these markers have also been applied to polyploid hybrids, (e.g., Janko et al. 2012; Mishina et al. 2014), when triploids were typically inferred by the possession of three peaks in at least one locus. However, the identification method proposed in this study suggests that microsatellite markers can be used as a powerful method to determine triploids even in cases when hybrids possess no more than two alleles, relying on the relative amplification intensities of species-specific alleles.

In a summary, the cross-species amplification of microsatellite markers can be used as an easy and fast identification method in studies of reproductive modes of investigated hybrids.

\section{Acknowledgments}

This project has been supported by the Czech Science Foundation grant No. 17-09807S and the Ministry of Education, Youth and Sports of the Czech Republic, Grant/ Award Number: EXCELLENCE CZ.02.1.01/0.0/0.0/ $15 \_003 / 0000460$ OP RDE.

\section{References}

Anonymous (2006) Ethical justification for the use and treatment of fishes in research. Journal of Fish Biology 68(1): 1-2. https://doi. org/10.1111/j.0022-1112.2006.01035.x

Anonymous (2011a) Development of culture techniques and construction of monitoring system for released seedings of an endangered fish Koreocobitis naktongensis. Ministry of Environment, South Korea. Soonchunhyang University, South Korea [In Korean]

Anonymous (2011b) Guide for the care and use of laboratory animals, 8th edn. National Research Council (NRC) National Academy Press, Washington, DC, USA.

Bang IC, Kim WJ, Lee IR (2009) Characterization of polymorphic microsatellite loci in the endangered Miho spine loach (Iksookimia choii) and cross-species amplification within the Cobitidae family. Molecular Ecology Resources 9(1): 281-284. https://doi. org/10.1111/j.1755-0998.2008.02452.x

Beacham TD, Le KD, Raap MR, Hyatt K, Luedke W, Wither R (2000) Microsatellite DNA variation and estimation of stock composition of Barkley Sound, British Columbia sockeye salmon. Fish Bulletin 98(1): 14-24.

Bohlen J, Šlechtová V (2009) Phylogenetic position of the fish genus Ellopostoma (Teleostei: Cypriniformes) using molecular genetic data. Ichthyological Exploration of Freshwaters 20(2): 157-162. de Gelas K, Janko K, Volckaert FAM, Charleroy DDE, Van Houdt JKJ (2008) Development of nine polymorphic microsatellite loci in the spined loach, Cobitis taenia, and cross-species amplification in the related species $C$. elongatoides, $C$. taurica and $C$. tanaitica. Molecular Ecology Resources 8(5): 1001-1003. https://doi.org/10.1111/ j.1755-0998.2008.02135.x

Goldstein DB, Schlötterer C (1999) Microsatellites: Evolution and Applications. Oxford University Press, New York, 368 pp.

Gonzalez-Martinez SC, Robledo-Arnuncio JJ, Collada C, Diaz A, Williams CG, Alia R, Cervera MT (2004) Cross-amplification and sequence variation of microsatellite loci in Eurasian hard pines. Theoretical and Applied Genetics 109(1): 103-111. https://doi. org/10.1007/s00122-004-1596-x

Janko K, Vasil'ev VP, Rab P, Rabova M, Slechtova V, Vasil'eva ED (2005) Genetic and morphological analyses of 50-chromosome spined loaches (Cobitis, Cobitidae, Pisces) from the Black Sea basin that are morphologically similar to $C$. taenia, with the description of a new species. Folia Zoologica 54(4): 405-420.

Janko K, Flajšhans M, Choleva L, Bohlen J, Šlechtová V, Rábová M, Lajbner Z, Šlechta V, Ivanova P, Dobrovolov I, Culling M, Persat H, Kotusz J, Ráb P (2007) Diversity of European spined loaches (genus Cobitis L.): An update of the geographic distribution of the 
Cobitis taenia hybrid complex with a description of new molecular tools for species and hybrid determination. Journal of Fish Biology 71(sc): 387-408. https://doi.org/10.1111/j.1095-8649.2007.01663.x

Janko K, Pačes J, Wilkinson-Herbots H, Costa RJ, Roslein J, Drozd P, Iakovenko N, Rídl J, Hroudová M, Kočí J, Reifová R, Šlechtová V, Choleva L (2018) Hybrid asexuality as a primary postzygotic barrier between nascent species: On the interconnection between asexuality, hybridization and speciation. Molecular Ecology 27(1): 248-263. https://doi.org/10.1111/mec.14377

Kim IS, Lee JH (1990) Diploid-triploid hybrid complex of the spined loach Cobitis sinensis and C. longicorpus (Pisces, Cobitidae). Korean Journal of Ichthyology 2(2): 203-210.

Kim IS, Lee EH (2000) Hybrization experiment of diploid-triploid cobitid fishes, Cobitis sinensis-longicorpus complex (pisces Cobitidae). Folia Zoologica 49(1): 17-22.

Kim IS, Park JY (2002) Freshwater fishes of Korea. Kyo-Hak Publishing, Seoul, 465 pp. [In Korean]

Ko MH (2009) Reproductive mechanisms of the unisexual diploid-tripoid hybrid complex of between the spined loach Cobitis hankugensis and Iksookimia longicorpa (Teleostei, Cobitidae) in Korea. Chonbuk National University, Doctoral thesis, 160 pp. [In Korean]

Lee EH (1995) A study of reproductive mode of the unisexual Cobitid fishes, Cobitis sinensis-longicorpus complex (Cobididae) by hybridization with its parental species. Chonbuk National University, Doctoral thesis, $92 \mathrm{pp}$. [In Korean]

Li CY, Li JT, Kuang YY, Xu R, Zhao ZX, Hou GY, Liang HW, Sun XW (2014) The transcriptomes of the crucian carp complex (Carassius auratus) provide insights into the distinction between unisexual triploids and sexual diploids. International Journal of Molecular Sciences 15(6): 9386-9406. https://doi.org/10.3390/ijms15069386

McConnell SK, O'eilly P, Hamilton L, Wrigh JM, Bentzen P (1995) Polymorphic microsatellite loci from Atlantic salmon (Salmo salar): Genetic differentiation of North American and European populations. Canadian Journal of Fisheries and Aquatic Sciences 52(9): 1863-1872. https://doi.org/10.1139/f95-779

Mishina T, Takada M, Takeshima H, Nakano M, Ryoichi T, Mutsumi N, Katsutoshi W (2014) Molecular identification of species and ploidy of Carassius fishes in Lake Biwa, using mtDNA and microsatellite multiplex PCRs. Ichthyological Research 61(2): 169-175. https:// doi.org/10.1007/s10228-014-0388-9

Moore SS, Sargeant SS, King TJ, Mattick JS, Georges M, Hetzel DJ (1991) The conservation of dinucleotide microsatellites among mammalian genomes allows the use of heterologous PCR primer pairs in closely related species. Genomics 10(3): 654-660. https:// doi.org/10.1016/0888-7543(91)90448-N

Nelson RJ, Beacham TD, Small MP (1998) Microsatellite analysis of the population structure of a Vancouver Island sockeye salmon
(Oncorhynchus nerka) stock complex using nondenaturing gel electrophoresis. Molecular Marine Biology and Biotechnology 7(4): 312-319.

Peakall R, Gilmore S, Keys W, Morgante M, Rafalski A (1998) Cross-species amplification of soybean (Glycine max) simple sequence repeats within the genus and other legume genera: Implications for the transferability of SSRs in plants. Molecular Biology and Evolution 15(10): 1275-1287. https://doi.org/10.1093/oxfordjournals.molbev.a025856

Senan S, Kizhakayil D, Sasikumar B, Sheeja TE (2014) Methods for development of microsatellite markers: An overview. Notulae Scientia Biologicae 6(1): 1-13. https://doi.org/10.15835/nsb619199

Šlechtová V, Bohlen J, Perdices A (2008) Molecular phylogeny of the freshwater fish family Cobitidae (Cypriniformes: Teleostei): Delimitation of genera, mitochondrial introgression and evolution of sexual dimorphism. Molecular Phylogenetics and Evolution 47(2): 812-831. https://doi.org/10.1016/j.ympev.2007.12.018

Smith CT, Koop BF, Nelson RJ (1998) Isolation and characterization of coho salmon (Oncorhynchus kisutch) microsatellites and their use in other salmonids. Molecular Ecology 7(11): 1613-1621.

Squirrell J, Hollingsworth PM, Woodhead M, Russell J, Lowe AJ, Gibby M, Powell W (2003) How much effort is required to isolate nuclear microsatellites from plants? Molecular Ecology 12(6): 1339-1348. https://doi.org/10.1046/j.1365-294X.2003.01825.x

Sunnucks P (2000) Efficient genetic markers for population biology. Trends in Ecology and Evolution 15(5): 199-203. https://doi. org/10.1016/S0169-5347(00)01825-5

Thiel T, Michalek W, Varshney RK, Graner A (2003) Exploiting EST databases for the development and characterization of gene-derived SSR-markers in barley (Hordeum vulgare L.). Theoretical and Applied Genetics 106(3): 411-422. https://doi.org/10.1007/s00122002-1031-0

Tian C, Guo W, Liang XF, Sun L, Lv L, Zhao C, Song Y, He S (2017) Identification of species-specific microsatellite markers in three Siniperca species by RNA-Seq. Biochemical Systematics and Ecology 70: 126-131. https://doi.org/10.1016/j.bse.2016.10.006

You C, Yu X, Tong J (2007) Detection of hybridization between two loach species (Paramisgurnus dabryanus and Misgurnus anguillicaudatus) in wild populations. Environmental Biology of Fishes 86(1): 65-71. https://doi.org/10.1007/978-90-481-3458-8_10

Yu JN, Kim BJ, Yun YE, Kim HS, Bae CH, Kim SN, Oh KH, Lim CE (2014) Characterization of 11 novel polymorphic microsatellite loci in the threatened Korean loach, Iksookimia koreensis, isolated using a next-generation sequencing method. Biochemical Systematics and Ecology 56: 132-137. https://doi.org/10.1016/j.bse.2014.05.016

Zane L, Bargelloni L, Patarnello T (2002) Strategies for microsatellite isolation: A review. Molecular Ecology 11(1): 1-16. https://oi. org/10.1046/j.0962-1083.2001.01418.x 